\title{
Detectores Multiusuários Adaptativos para DS-CDMA
}

\section{Adaptive Multiuser Detectors for DS-CDMA Systems}

\author{
Fernando Ciriaco $^{1}$; Lisiane Heringer ${ }^{2}$; Luis Carlos Albuquerque ${ }^{3}$; Taufik \\ Abrão ${ }^{4}$; Paul Jean E. Jeszensky ${ }^{5}$
}

\section{Resumo}

Este trabalho faz uma revisão dos principais detectores multiusuários adaptativos ((MuD-Adpt) subótimos para sistemas DS-CDMA (Direct Sequence - Code Division Multiple Access). São enfocados os detectores adaptativos baseados no erro quadrático médio mínimo (MMSE - Minimum Mean Square Error) e os de descorrelação (MuD-Dec). Detectores multiusuários apresentam ótima resistência ao efeito perto-longe (near-far effect) e combatem efetivamente a interferência de múltiplo acesso (MAI). Resultados numéricos comparativos caracterizam o aumento substancial de desempenho desses detectores adaptativos em relação ao receptor convencional com filtro casado (Conv).

Palavras-chave: DS-CDMA, Detecção Multiusuário, Descorrelacionador, MMSE, Algoritmos de Detecção Adaptativos, Convergência, 3G.

\begin{abstract}
This work makes a review of the main Adaptives Multi-user Detectors (MuD-Adpt) for Direct Sequence Code Division Multiple Access (DS-CDMA) systems. The MuD-Adpt based on Minimum Mean Square Error (MMSE) and Decorrelator (MuD-Dec) are focused. Multi-user detectors show great resistance to the near-far effect and combat effectively the Multiple Access Interference (MAI). Comparative numeric results characterize the substantial performance improvement of those detectors in relation to the matched filter conventional receiver (Conv).

Keywords: DS-CDMA, Multi-user Detection, Decorrelator, MMSE, Adaptive Detection Algorithms, Convergence, $3 \mathrm{G}$.
\end{abstract}

${ }^{1,2,3}$ Alunos de mestrado, Departamento de Engenharia Elétrica, Universidade Estadual de Londrina (DEEL-UEL)

Professor Adjunto do DEEL-UEL; taufik@uel.br

Professor Titular da Escola Politécnica da USP, PTC; pjj@lcs.poli.usp.br 


\section{Introdução}

O detector convencional baseado no filtro casado resulta em uma capacidade sistêmica bem abaixo da capacidade do canal, além de sofrer a limitação de não ser resistente ao efeito near-far, requerendo cuidadoso e preciso controle de potência. Assim, estudos realizados nos últimos quinze anos têm buscado novos algoritmos de detecção multiusuário (MuD) objetivando a redução ou mesmo a eliminação da interferência de múltiplo acesso (MAI). Versões MuD subótimas fixas foram tratadas em (ABRÃO; JESZENSKY, 2001a) (ABRÃO; JESZENSKY, 2001b).

Abordagens adaptativas para a técnica de detecção multiusuário subótima para sistemas de múltiplo acesso por divisão de código (CDMA - Code Division Multiple Access) prometem aumento de desempenho e de capacidade por meio da atualização contínua das estimativas dos parâmetros de canal. Basicamente, existem três formas de implementação desses algoritmos: descorrelacionador, erro quadrático médio mínimo (MMSE - Minimum Mean Square Error) e cancelamento de interferência adaptativos (VUCETIC; WOODWARD, 1998; ZVONAR; DUEL-HALLEN，1995; MOSHAVI， 1996; VERDÚ, 1998). Sob o ponto de vista da necessidade de conhecimento das informações dos usuários interferentes, as estruturas MMSE adaptativas não apresentam imposições para atingir desempenho aceitável; o descorrelacionador adaptativo pressupõe um conhecimento razoavelmente preciso dos parâmetros de sistema e as estruturas de cancelamento de interferência (IC) adaptativas necessitam um acurado conhecimento dos parâmetros dos interferentes mais significativos.

Detectores adaptativos são realizações aproximadas de estruturas receptoras multiusuário fixas. Nos detectores multiusuários subótimos subtrativos, são geradas estimativas de interferência MAI e posteri- ormente subtraídas do sinal desejado. As estruturas receptoras baseadas no cancelamento de interferência sucessivo e paralelo, SIC e PIC (Successive e Parallel Interference Cancellation), respectivamente, e o ZF-DF (Zero Forcing Decision Feedback) pressupõem a explícita detecção seguida do cancelamento de cada sinal de usuário a partir dos demais. Tais técnicas têm como pré-requisito o conhecimento acurado dos parâmetros de canal e podem, em certas condições de operação do sistema, resultar em excessiva complexidade computacional.

Em (LIN; LEE; TAN, 2002) foi proposto um receptor parcialmente adaptativo, visando a incrementar a supressão da MAI. Na ausência de informação do canal, a supressão eficaz da interferência é conseguida construindo-se um conjunto de canceladores de lóbulo lateral generalizado (Generalized SideLobe Cancellation), associado à técnica do gradiente conjugado. Zhu et al (ZHU; ARASARATNAM; CONSTANTINIDES, 2002) propuseram um receptor DS-CDMA adaptativo cego para ambientes de canal multipercursos práticos baseado na separação das componentes multipercursos, com identificação usuário-e-atraso. Já em (MAROUANE; KACHOURI; KAMOUN, 2004) propôs-se uma abordagem de detecção single-user adaptativa que utiliza um filtro adaptativo baseado no critério do mínimo erro quadrático médio (LMS - Least Mean Squared), capaz de combater a MAI e combinar coerentemente os multi-percursos.

Resultados recentes da literatura sobre MuDAdapt incluem (MUCCHI et al., 2004), (LI; HAMOUDA, 2005), (TAKAWIRA, 2005), (SUN; BI; ZHANG, 2004), (KARAYIANNIS; CHOOKIARTI, 2005), (ZHANG; D'AMOURS; YONGACOGLU, 2005), (NIEKERK; MNENEY, 2004). Uma versão modificada para o detector multiusuário adaptativo cego DS-CDMA (Direct Sequence - CDMA) é proposta em (MUCCHI et al., 2004). Já em (LI; HAMOUDA, 2005) os autores analisam um esquema de detecção multi- 
estágio de baixa complexidade para sistemas DSCDMA capaz de tratar o sinal com desvanecimento plano e seletivo em freqüência a partir da técnica adaptativa cego no primeiro estágio. $\mathrm{O}$ autor de (TAKAWIRA, 2005) quantifica o desempenho de um detector multiusuário adaptativo cego baseado no algoritmo de módulo constante ajustado linearmente (LCCMA), tanto em um canal estacionário como em não-estacionário. Já em (SUN; BI; ZHANG, 2004) os autores utilizam o LCCMA no desenvolvimento de um detector multiusuário adaptativo cego de convergência rápida, também para sistemas DS-CDMA. Em (KARAYIANNIS; CHOOKIARTI, 2005) foram desenvolvidos detectores adaptativos com estimativas diretas (DEA) para sistemas CDMA. (ZHANG; D'AMOURS; YONGACOGLU, 2005) aplicaram a técnica adaptativa para detecção multiusuário SISO (soft-input soft-output), evitando assim a necessidade da obtenção da informação a priori. Um tutorial recente sobre detecção multiusuário adaptativo para sistemas CDMA pode ser encontrado em (NIEKERK; MNENEY, 2004); primeiramente o foco é dado aos detectores adaptativos cegos; discute-se em seguida questões relativas à codificação de erro aplicado a sistemas de CDMA. Particular atenção é dada à codificação turbo introduzindo-se a possibilidade de se ter um decodificador turbo combinado à arquitetura do detector CDMA.

Deve-se ressaltar a existência de problemas práticos e tópicos de pesquisa ainda abertos, tipicamente associados à taxa de convergência e desempenho de sincronismo dos algoritmos adaptativos, particularmente aplicáveis à detecção multiusuário DS/CDMA.

O modelo de sistema DS/CDMA empregado neste trabalho, baseado em canal com ruído branco Gaussiano aditivo (AWGN - Additive White Gaussian Noise) e modulação BPSK (PROAKIS, 1989), foi des- crito em (ABRÃO; JESZENSKY, 2001a) e não será reproduzido aqui. Na próxima seção são analisadas as principais topologias utilizadas nos detectores MuDAdapt de descorrelação e MMSE, além de aspectos relacionados à convergência e sincronismo desses algoritmos. Em seguida, são analisados resultados numéricos de convergência e desempenho. Na última seção são destacadas as principais conclusões desse trabalho.

\section{Detectores Multiusuários Adaptati- VOS}

Em detectores lineares não adaptativos (MMSE e Descorrelacionador Fixos), faz-se necessário o cômputo da resposta impulsiva do filtro, envolvendo inversões das matrizes de correlação cruzada. São necessárias intensas operações de inversão dessas matrizes quando os canais são assíncronos e suas correlações cruzadas são variantes no tempo. O mesmo se verifica em canais nos quais as potências recebidas sejam variantes no tempo (no caso do receptor MMSE fixo linear multiusuário). Assim, torna-se altamente desejável obter detectores multiusuário lineares que não só eliminem a necessidade do cômputo on-line da resposta impulsiva, mas também não se utilize do conhecimento das correlações cruzadas ou das seqüências de espalhamento dos usuários interferentes. Isto pode ser obtido a partir da versão adaptativa do receptor MMSE, o qual é capaz de construir a desejada resposta impulsiva do filtro a partir da forma de onda recebida, pressupondo que os dados do usuário de interesse sejam conhecidos no receptor. Isto implica a transmissão de uma sequiência de treinamento que consiste de uma seqüência de dados previamente conhecida no receptor. O receptor utiliza um padrão adaptativo para ajustar a transformação linear enquanto a sequiência de treinamento está sendo transmitida. Finalmente, caso as correlações cruzadas e ampli- 
tudes mudem com o tempo, a seqüência de treinamento pode ser enviada periodicamente para reajustar os parâmetros do receptor. Na prática, é comum realizar ajustes "finos" da transformação linear (uma vez que o algoritmo tenha convergido e a transmissão da seqüência de treinamento tenha sido finalizada), deixando que o algoritmo adaptativo opere com as próprias decisões realizadas pelo detector ao invés das informações contidas na seqüência de treinamento. A este procedimento denomina-se decisão direcionada. A única informação necessária no receptor para se obter a equalização adaptativa MMSE é a seqüência de treinamento para o usuário de interesse. O sincronismo é requerido apenas em nível de símbolo, dependo do número de estágios (time span) do filtro receptor. A amostragem à taxas de sub-chip permite reduzir os requisitos de sincronismo.

A imposição do requisito seqüência de treinamento em receptores adaptativos implica algum tipo de overhead de sistema, equivalente a um tom piloto ou sequiência preâmbulo em uma abordagem utilizando estimação de canal. Para evitar este tipo de overhead, os trabalhos (HONIG; MADHOW; VERDÚ., 1995) (MADHOW, 1998) (WANG; POOR, 1998b) (ULUKUS; YATES, 1998) (WANG; POOR, 1998a) (TONG; PERREAU, 1998) exploram técnicas cegas de equalização a fim de possibilitar a eliminação da sequiência de treinamento. Isso temse mostrado possível, porém com alguma restrição no grau de distorção introduzida pelo canal. A técnica, todavia, tem apresentado bons resultados na eliminação da interferência banda estreita (NBI) e simultaneamente na supressão da interferência MAI.

\section{Detector Multiusuário de Descorrelação Adaptativo}

O receptor multiusuário de Descorrelação multiplica a saída de um banco de filtro casados pela inversa da matriz de correlação cruzada, (32) em (ABRÃO;
JESZENSKY, 2001a):

$$
\hat{\mathbf{b}}_{k}=\operatorname{sgn}\left(\mathbf{R}^{-1} \mathbf{y}\right)
$$

onde $\hat{\mathbf{b}}_{k}$ é a estimativa dos dados, $\mathbf{R}$ é a matriz de correlação (auto e cruzada) e y é o vetor saída de um banco de filtro casados. Chen e Roy (CHEN; ROY., 1994) mostraram como realizar de forma adaptativa o detector de Descorrelação; propuseram o filtro para a reconstrução do sinal da Fig. 1 objetivando a obtenção da descorrelação dos sinais. Como desvantagem, há aumento de ruído em relação ao Descorrelacionador fixo. A abordagem consiste em encontrar o vetor de coeficientes, o qual multiplica as sequiências de espalhamento para formar uma boa estimativa para o sinal recebido. Assume-se canal AWGN. No início de cada intervalo de símbolo, os coeficientes são anulados e então atualizados a cada intervalo de amostragem.

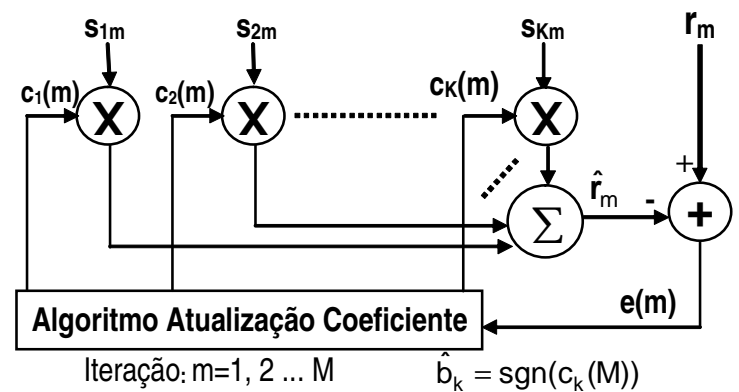

Figura 1: Receptor de descorrelação adaptativo, usuário $k$

Os coeficientes ótimos do filtro transversal para a $n$-ésima iteração pode ser computada de acordo com:

$$
c(n)=\mathbf{R}^{-1}(n) \mathbf{D}(n)
$$

onde, empregando a abordagem de mínimos quadrados, a matriz de correlações amostrada pode ser determinada iterativamente por (HAYKIN, 1996):

$$
\mathbf{R}(n)=\sum_{m=1}^{n} \lambda^{n-m} \mathbf{S}_{m}^{H} \mathbf{S}_{m}
$$




$$
\mathbf{D}(n)=\sum_{m=1}^{n} \lambda^{n-m} r_{m} \mathbf{S}_{m}^{H}
$$

onde $\mathbf{S}_{m}$ é o vetor observação (no contexto $\mathrm{MuD}$, o código de espalhamento), $r_{m}$ é a resposta desejada e $\lambda=$ constante de decaimento da memória (ou fator de esquecimento), com valores no intervalo $[0,1]$. Valores de $\lambda<1$ permitem obter sincronismo em canais não estacionários: quanto menor o valor de $\lambda$, maior a adaptabilidade às mudanças do canal. Os valores finais para $\mathbf{c}$ do filtro ótimo são:

$$
\mathbf{c}(M)=\sqrt{\mathbf{E}} \mathbf{b}+\mathbf{R}(M)^{-1} \boldsymbol{\Gamma}
$$

onde $\mathbf{E}=\operatorname{diag}\left\{E_{1}, E_{2}, \ldots, E_{K}\right\}$ é a matriz energia recebida, $\boldsymbol{\Gamma}=\mathbf{S}^{H} \mathbf{n}$ é um processo Gaussiano de média zero e $M$ é o número de amostras por símbolos. A expressão (5) indica a versão adaptativa para o detector de Descorrelação em (ABRÃO; JESZENSKY, 2001a). Decisões são feitas de acordo com os sinais das derivações do filtro ao final de cada período de símbolo,

$$
\hat{\mathbf{b}}=\operatorname{sgn}[\mathbf{c}(M)]
$$

com média $\overline{\mathbf{c}}=\sqrt{\mathbf{E}} \mathbf{b}$ e variância de cada coeficiente dada pelos elementos da diagonal da matriz de covariância:

$$
\sigma_{c}^{2}=\left[\sigma_{c 1}^{2} \sigma_{c 2}^{2} \sigma_{c 3}^{2} \ldots . \sigma_{c K}^{2}\right]^{H}=\sigma_{n}^{2} \operatorname{diag}\left(\mathbf{R}^{-1}\right)
$$

Assume-se independência das amostras do ruído. A taxa de erro de bit (BER) para o $k$-ésimo usuário à saída do Descorrelacionador adaptativo em canal AWGN será:

$$
P_{k}^{D e c A d p}=Q\left(\frac{A_{k}}{\sigma_{c_{k}}}\right)
$$

onde $A_{k}$ representa a amplitude recebida do $k$-ésimo usuário e $Q(\cdot)$ está relacionado à função erro complementar, com $Q(x)=\frac{1}{2 \pi} \int_{x}^{\infty} e^{\frac{-u^{2}}{2}} d u=$ $\frac{1}{2}$ erf $c\left(\frac{x}{\sqrt{2}}\right)$. Uma possível alternativa ao esquema adaptativo de Chen e Roy (CHEN; ROY., 1994) foi proposto em (MYERS; MAGANA, 1997) para o cálculo dos coeficientes do detector de Descorrelação sem a necessidade de inversões de matrizes. A técnica está baseada em um eficiente cálculo de ortogonalização de Gram-Schmidt para todos os vetores de espalhamento.

Observe-se que o detector de descorrelação adaptativo deve obter estimativas de dados a cada intervalo de bit, $T_{b}$. Neste intervalo, o detector MuDAdapt deve reconstruir o sinal transmitido a partir do sinal recebido e das seqüências dos usuários. É comum iniciar as estimativas para os coeficientes do filtro um vetor aleatório binário bipolarizado $( \pm 1)$.

Visando a garantir convergência do algoritmo Decor-Adapt para diferentes condições de operação do sistema como relação sinal-ruído $S N R$, número de usuários ativos $K$, efeito near-far, entre outros, é comum adotar a seguinte regra prática para o parâmetro passo de convergência:

$$
\mu=\frac{\mu_{\max }}{10}
$$

sendo $\mu_{\max } \mathrm{o}$ maior valor encontrado na etapa de treinamento. A vantagem desse método é que não é necessário uma etapa de treinamento. No entanto, para ser possível estimar o símbolo transmitido do $k$-ésimo usuário com acurácia, deve-se realizar um número de iterações em tempo real da ordem de 200, quando o sistema estiver operando em canais AWGN e carregamento médio, podendo resultar em um tempo de processamento maior que períodos de bit típicos para aplicações multimídia, acarretando na impossibilidade de implementação.

\section{Detector Multiusuário MMSE Adaptativo}

O detector MMSE adaptativo é empregado quando os parâmetros dos interferentes são desconhecidos 
e/ou variantes no tempo. O número de coeficientes em um receptor MMSE está relacionado ao ganho de processamento e não ao número de usuários ativos. Processando diretamente amostras do sinal recebido em intervalos de chip (ou sub-chip), o receptor MMSE adaptativo opera como um equalizador adaptativo. Compreende um filtro que simultaneamente está casado com o sinal do usuário desejado, suprimindo a interferência dos demais usuários através da minimização do erro quadrático médio (MSE - Mean Square Error). Tal minimização pode ser realizada sobre todos os usuários ou para cada usuário individualmente. Pode-se mostrar que as duas abordagens são equivalentes (VERDÚ, 1998). As componentes do custo MSE a serem minimizadas incluem os efeitos da MAI, da interferência intersimbólica (ISI), do ruído AWGN e, às vezes, da NBI.

Com um adequado número de estágios no filtro adaptativo, o receptor MMSE adaptativo atua como um receptor RAKE (HONIG; MADHOW; VERDÚ., 1995), combinando energia de todas as componentes multipercursos de um sinal de usuário, sem a necessidade de complexidade extra para tratar separadamente a sincronização de cada um dos multipercursos. Como o receptor MMSE linear fixo, definido pela transformação linear (ABRÃO; JESZENSKY, 2001a)

$$
\mathbf{T}=\left[\mathbf{R}+\sigma^{2} \mathbf{A}^{-2}\right]^{-1}
$$

requer o conhecimento da matriz de correlação normalizada, das amplitudes do sinais, da potência de ruído e um banco de filtro casado sincronizados, acaba por sofrer do problema de muitos receptores multiusuários que consiste em assumir o conhecimento de demasiada quantidade de parâmetros. Adicionalmente, nos casos nos quais o canal não for estacionário, far-se-ão inúmeras e repetidas inversões de matrizes, resultando em elevado custo computacional. No entanto, o detector MMSE pode ser aproximado por um filtro de resposta impulsiva (FIR) adap- tativo, sendo tratado exatamente como um problema de equalização adaptativa com um critério de erro MSE. Neste caso, a transformação linear para o receptor MMSE adaptativo torna-se:

$$
\mathbf{T}=\left[\mathbf{Q}+\sigma^{2} \mathbf{I}_{K}\right]^{-1}
$$

com $\mathbf{I}_{K}$ matriz identidade de dimensão $K$, a ma$\operatorname{triz} \mathbf{Q}=\sqrt{\mathbf{E}} \mathbf{H} \sqrt{\mathbf{E}}, \mathbf{H}=\mathbf{P}^{H} \mathbf{P}$ e $\mathbf{P}=$ $\left[\mathbf{p}_{1}, \mathbf{p}_{2}, \ldots, \mathbf{p}_{K}\right]$, com $\mathbf{p}_{k}$ o código de espalhamento que identifica o $k$-ésimo usuário em um sistema DS-CDMA coerente em canal AWGN (ABRÃO; JESZENSKY, 2001a). Nele [.] ${ }^{H}$ representa o operador matricial Hermitiano, i.e., o complexo conjugado transposto.

Uma importante característica no receptor MMSE adaptativo é que o critério MSE pode ser minimizado para cada usuário individualmente:

$$
\hat{\mathbf{b}}_{k}=\operatorname{sgn}\left(\mathbf{c}_{k}^{H} \mathbf{r}\right)
$$

onde os coeficientes do filtro linear MMSE $\mathbf{c}_{k}$ podem ser obtidos a partir de duas formas:

$$
\begin{aligned}
\mathbf{c}_{k} & =\left(\mathbf{Q}+\sigma_{n}^{2} \mathbf{I}\right)^{-1} \sqrt{E_{k}} \mathbf{p}_{k} \\
& =\mathbf{P}\left[\left(\mathbf{H}+\sigma_{n}^{2} \mathbf{I}_{K}\right)^{-H}\right]_{k}
\end{aligned}
$$

onde $[\cdot]_{k}$ representa a extração da $k$-ésima linha da matriz. A última igualdade em (11) pode ser mostrada empregando-se identidade de Woodbury (lema da inversão de matriz, (HAYKIN, 1996)). A resistência ao efeito near-far para o detector MMSE linear adaptativo é a mesma do MMSE fixo, (27) em (ABRÃO; JESZENSKY, 2001a); para o primeiro usuário tem-se:

$$
\bar{\eta}_{1}^{M M S E a d a p t}=1-\mathbf{a}_{1}^{T} \mathbf{R}_{1}^{-1} \mathbf{a}_{1}
$$

A saída do filtro MMSE adaptativo é (ABRÃO; JESZENSKY, 2001a) 


$$
\begin{array}{cc}
z_{k}=\mathbf{t}_{k}^{H} \mathbf{R} \sqrt{\mathbf{E}} \mathbf{b}+\mathbf{t}_{k}^{H} \mathbf{P}^{H} \mathbf{n} \\
\text { com média } & \bar{z}_{k}=\mathbf{t}_{k}^{H} \mathbf{R} \sqrt{\mathbf{E}} \mathbf{b} \\
\text { e variância } & \sigma_{z_{k}}^{2}=\sigma_{n}^{2} \mathbf{t}_{k}^{H} \mathbf{R} \mathbf{t}_{k}
\end{array}
$$

Para encontrar a BER, é necessário somar a cauda da função Gaussiana sobre todos os possíveis vetores de dados empregando-se a média e variância acima. Isto resulta em um cálculo dispendioso. Uma aproximação pode ser derivada caracterizando a distribuição $z_{k}$, condicionada a $b_{k}=1$. Assumindose que os dados do usuário de interesse tenham média zero, sejam independentemente distribuídos e tomando o teorema do limite central, $z_{k}$ pode ser considerada uma variável aleatória com distribuição Gaussiana. A saída do filtro terá média e variância dadas por:

média: $\quad \bar{z}_{k}=\mathbb{E}\left[z_{k} \mid b_{k}=1\right]=1-J_{\min _{k}}$

$$
\begin{array}{ll}
\text { variância: } \quad & \sigma_{z_{k}}^{2}=\mathbb{E}\left[\left(z_{k}-\bar{z}_{k}\right)^{2} \mid b_{k}=1\right] \\
& =\bar{z}_{k}-\bar{z}_{k}^{2}=J_{\min _{k}}\left(1-J_{\min _{k}}\right)
\end{array}
$$

onde $\mathbb{E}[\cdot]$ é o operador esperança estatística, com $J_{\min _{k}}=$ mínimo valor para o erro MSE, usuário $k$, dado por:

$$
\begin{gathered}
J_{\min _{k}}=1-\alpha_{k}^{H}\left(\mathbf{Q}+\sigma_{n}^{2} \mathbf{I}\right)^{-1} \alpha_{k} \\
\text { onde } \quad \alpha_{k}=\sqrt{E_{k}} \mathbf{p}_{K}
\end{gathered}
$$

A aproximação Gaussiana para o desempenho de BER do usuário $k$ de um receptor MMSE adaptativo ideal é:

$$
P_{k}^{\substack{M M S E \\ \text { Adpt Assinc }}} \approx Q\left(\sqrt{\frac{1-J_{\min _{k}}}{J_{\min _{k}}}}\right)
$$

Uma pequena modificação no MMSE adaptativo deve ser feita na abordagem seguida até aqui para contemplar canal AWGN assíncrono. O receptor adaptativo necessita conhecer os dados do usuário de interesse e sincronizá-lo ao nível de bit. Para um sistema DS-CDMA assíncrono, Miller (MILLER, 1995), assumindo hipótese Gaussiana, centrada sobre os pontos de sinal em $b_{k} \in\{-1,1\}$, obteve:

$$
P_{k}^{\substack{M M S E \\ \text { Adpt Assinc }}} \approx Q\left(\sqrt{\frac{1}{J_{\min _{k}}}}\right)
$$

A resistência ao efeito near-far do detector MMSE linear one-shot (VERDÚ, 1998) é a mesma do detector de descorrelação one-shot, mas não é tão bom quanto do detector de descorrelação assíncrono (VERDÚ, 1998). Note-se que para baixas SNR a média da distribuição Gaussiana é bastante próxima de zero e, portanto, a BER é maior no caso assíncrono. O filtro MMSE pode ser aproximado adaptativamente por muitos algoritmos (HAYKIN, 1996). Alguns desses algoritmos serão vistos rapidamente a seguir.

\section{Algoritmo LMS para o Detector MMSE}

O algoritmo de mínimo erro quadrático médio (LMS) aproxima o método declive máximo (steepest descent) (HAYKIN, 1996). Opera tomando passos pequenos sobre a superfície de desempenho do erro quadrático em direção ao gradiente negativo. Emprega-se a estimação de gradientes instantâneos. Os coeficientes são atualizados seguindo:

$$
\begin{aligned}
\hat{c}_{k}(n+1) & =\hat{c}_{k}(n)-\mu \nabla\left[\left|e_{k}(n)\right|^{2}\right] \\
& =\hat{c}_{k}(n)+\mu E\left[\mathrm{r}_{k}(n) e_{k}^{*}(n)\right] \\
& \approx \hat{c}_{k}(n)+\mu \mathrm{r}_{k}(n) e_{k}^{*}(n)
\end{aligned}
$$

onde, $n=$ tempo discreto; $\mu=$ dimensão do passo, limitado pelos requisitos conflitantes: operação estável e velocidade de convergência aceitável. Assumindo-se conhecido o bit a ser detectado, o erro 
será dado por:

$$
e_{k}(n)=b_{k}(n)-z_{k}(n)
$$

onde $z_{k}(n)=\hat{c}_{k}(n) \mathrm{r}_{k}(n)$.

A Fig. 2 mostra a estrutura de um detector MMSE adaptativo LMS single-user realizado a partir do processamento direto do sinal recebido amostrado, resultando em uma estrutura simples. A implementação é feita através de uma linha de atraso e de mecanismo de atualização dos coeficientes do filtro a partir de derivações apropriadas. Como vantagem, o algoritmo não requer sincronização acurada e estágios de processamento multiusuário, os quais aumentam a complexidade do receptor DS/CDMA.

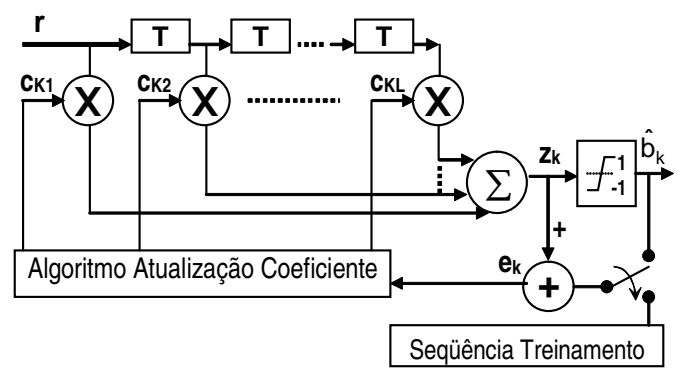

Figura 2: Receptor MMSE adaptativo single-user para o $k$-ésimo usuário baseado em filtro Transversal FIR

O detector MMSE adaptativo da Fig. 2 procura estimar o símbolo transmitido pelo $k$-ésimo usuário. Para isso, após convergência, os valores dos coeficientes do filtro devem resultar próximos aos valores de chip da seqüência de espalhamento. Adicionalmente, esses coeficientes também devem refletir os efeitos da MAI, near-far e do ruído AWGN. Verificou-se, por meio de simulação, que a adoção de valores aleatórios binários bipolarizados $( \pm 1)$ como estimativa inicial para os coeficientes melhora sensivelmente a velocidade de convergência do algoritmo em canal AWGN quando a SNR for elevada.

Alguns resultados preliminares indicaram uma alta sensibilidade do algoritmo ao parâmetro $\mu$. O incremento de valores para esse parâmetro, mesmo que modesto, resultou, em degradação de desempenho (em termos de BER). Optou-se por utilizar um valor de $\mu$ bem conservador, garantindo a estabilidade do algoritmo. Com isso, adotou-se o parâmetro $\mu=\mu_{\max } / 200$, sendo $\mu_{\max }$ o maior valor encontrado na etapa de treinamento, tal que ainda há convergência. A desvantagem desse método é a necessidade de uma etapa de treinamento, acarretando em diminuição da taxa líquida de informação transmitida. Além disso, para que seja possível estimar com uma certa acurácia o símbolo transmitido do $k$-ésimo usuário, deve-se realizar um número de iterações da ordem de 1000 na etapa de treinamento. Observese que o processo de treinamento para o detector MMSE adaptativo da Fig. 2 deve ser repetido para atender os $k$ usuários de interesse, pois trata-se de uma abordagem single-user.

Short, Xie and Rushforth, (XIE; SHORT; RUSHFORTH, 1990), aplicaram a abordagem MMSE linear adaptativa à estrutura multiusuário. Uma possível implementação para o receptor MMSE adaptativo multiusuário é mostrada na Fig.3. As saídas do banco de filtros casados são processadas por um filtro transversal FIR adaptativo. A desvantagem da abordagem multiusuário inclui aumento de complexidade e requisito do banco de correlatores sincronizados. Em algoritmos adaptativos, o uso de passos $\mu$, mesmo que pequenos (diferentes de zero) implica que os valores finais dos coeficientes calculados desviam-se aleatoriamente dos valores ótimos, ou seja, da solução de Wiener; portanto sempre resultam em algum desajuste no filtro adaptativo. O erro quadrático médio acima daquele obtido pelo custo MMSE é denominado excesso de erro quadrático médio. O desajuste no filtro adaptativo é dado por (HAYKIN, 1996):

$$
\mathcal{M}=\frac{J_{e x c}(\infty)}{J_{\min }}=\sum_{i=1}^{M} \frac{\mu \lambda_{i}}{2-\mu \lambda_{i}}
$$


onde $J_{\text {exc }}(\infty)$ é o excesso de MSE após convergência total; $\lambda_{i}$ é o $i$-ésimo autovalor da matriz de correlações $\mathbf{M}$, dimensão $M \times M$.

Em ambiente não estacionário, o retardo de sincronismo também resulta em excesso de erro MSE. Em ambiente com elevado ruído ou interferência faz-se necessário desativar a operação de decisão direcionada; caso contrário, pode ocorrer reversão à saída do filtro e as distribuições de probabilidade condicional não mais aproximam a média e variância.

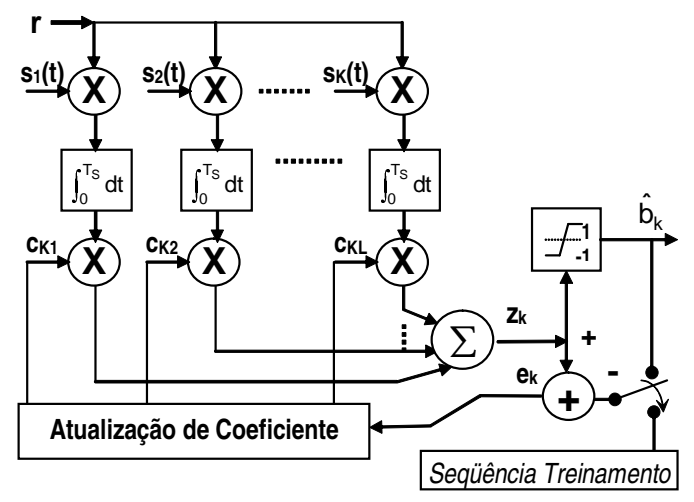

Figura 3: Detector MMSE adaptativo multiusuário, usuário $k$

O detector MMSE adaptativo multiusuário busca estimar o símbolo transmitido pelo $k$-ésimo usuário a partir de um banco de filtros casados às sequiências de espalhamento. Nesse caso, não há conhecimento prévio da região (valores) para os coeficientes. Dessa forma, utilizou-se como estimativa inicial para os coeficientes um vetor de zeros.

Para esse algoritmo com abordagem multiusuário, não há necessidade de se utilizar um valor conservador para o parâmetro $\mu$, diminuindo consideravelmente o número de iterações necessário à convergência do algoritmo. Com isso, nos resultados de simulação da seção adotou-se parâmetro $\mu=$ $\mu_{\max } / 10$.

Esse método também possui a desvantagem da necessidade de uma etapa de treinamento, acarretando em diminuição da taxa líquida de informação transmitida. No entanto, para que se possa estimar com acurácia o símbolo transmitido pelo $k$-ésimo usuário, deve-se realizar um número de iterações da ordem de 300 na etapa de treinamento e mesmas condições de operação que no algoritmo anterior, mostrando que essa técnica é mais vantajosa do que o método MMSE single-user discutido anteriormente.

O algoritmo LMS normalizado (NLMS) é uma variação do LMS, dado em (19), com vantagens de realização (HAYKIN, 1996). Nesta variante do algoritmo LMS, o valor do passo é obtido de forma dinâmica, de tal forma a ser o inverso da potência do sinal instantâneo:

$$
\mu=\frac{\bar{\mu}}{a+\|\mathbf{r}(n)\|^{2}}
$$

onde $\bar{\mu}=$ constante empregada no ajuste da velocidade de convergência; $a$ é a constante que garante a estabilidade quando a energia do sinal for pequena.

O filtro adaptativo requer um período inicial de treinamento, durante o qual o sinal erro empregado para atualização é gerado a partir da diferença entre a saída do filtro transversal FIR e uma cópia da seqüência transmitida, conhecida e armazenada no receptor. Após a fase de "aprendizado", os coeficientes podem ser mantidos constantes para um ambiente estacionário, ou continuamente adaptados às variações do canal no modo decisão direcionada. Nesta fase, o erro é formado a partir de:

$$
e_{k}(n)=\hat{b}_{k}(n)-\mathbf{c}_{k}^{H} \mathbf{r}
$$

Quando houver perturbações no canal, mudanças no número de usuários ativos (chegadas e partidas) ou mesmo alterações significativas nas potências dos sinais recebidos, um novo aprendizado será necessário.

Um filtro adaptativo proposto em (SLOCK, 1993) 
emprega técnicas de gradiente estocástico para alcançar a convergência.

\section{Convergência e Sincronismo do MuD MMSE Adaptativo}

O maior problema do receptor MMSE adaptativo consiste na inadequada velocidade de convergência e capacidade de sincronismo. Convergência lenta implica grandes períodos de treinamento e novos treinamentos, tempo em que não é possível enviar dados. A convergência da solução MMSE depende da estabilidade das amplitudes e correlações cruzadas. Quando estes parâmetros mudarem muito lentamente, relativamente à velocidade de convergência do algoritmo, é possível ainda seguir essas variações, quer através de seqüência de treinamento, quer no modo decisão direcionada. No entanto, caso haja uma mudança súbita no canal CDMA, como, por exemplo, a ativação de um usuário com potência elevada, o algoritmo de decisão direcionada será inicializado usando decisões não confiáveis, podendo não convergir. Faz-se necessário, então, que o receptor seja capaz de detectar tal mudança brusca e orientar o usuário a interromper sua transmissão de dados e dar lugar ao envio da sequiência de treinamento. Dois parâmetros afetam a velocidade de convergência do filtro LMS:

1. dimensão do filtro;

2. espalhamento dos autovalores da matriz de correlação

$$
\vartheta=\frac{\lambda_{\max }}{\bar{\lambda}_{\mathrm{eft}}}
$$

onde $\lambda_{\max }=$ máximo autovalor de $\mathbf{R}$ e $\bar{\lambda}_{\text {eft }}=$ autovalor médio efetivo; os autovalores insignificantes são desprezados.

As duas principais vantagens do algoritmo LMS são simplicidade e imunidade a ruído. A comple- xidade de implementação do algoritmo LMS é proporcional a $\mathcal{O}(S p)$, onde $p$ é o número de amostras/símbolo e $S$ é a quantidade de símbolos que o filtro processa.

\section{Algoritmo Mínimos Quadrados Recursivos, RLS para o Detector MMSE}

O algoritmo RLS (Recursive Least Squares) pode ser aplicado à estrutura linear MMSE, minimizando a função custo a partir da soma dos quadrados, (MILLER, 1995):

$$
J_{R L S}=\sum_{i=1}^{n} \lambda^{n-i}|e(i)|^{2}
$$

Tipicamente resulta em uma maior velocidade de convergência que o LMS. Além disso, a taxa de convergência é independente do espalhamento dos autovalores da matriz de correlação. Aqui, o parâmetro tamanho do passo, conhecido por $\mu$ no LMS, é definido pela inversa da matriz de correlação do vetor de entrada. Isto implica diferença na velocidade de convergência quando comparado ao LMS. O cálculo do erro quadrático médio é baseado em uma estimação de erro a priori:

$$
\xi(n)=b_{k}(n)-\hat{c}_{k}^{H}(n-1) r_{k}(n)
$$

O maior impedimento da utilização do algoritmo RLS é sua complexidade computacional, $\mathcal{O}\left(S^{2} p^{2}\right)$.

O algoritmo RLS padrão é bastante propenso a problemas de instabilidade numérica e de divergência (HAYKIN, 1996). Isto é de particular interesse em sistemas DS-CDMA com baixo AWGN e nos casos em que ocorre um número significativo de autovalores da matriz de correlação muito menor que a dimensão do filtro. Autovalores muito pequenos ficam próximo ao piso de AWGN e podem, então, causar mal condicionamento da matriz de correlação. 
Em (UENG; JENG, 2003) é apresentado um receptor de supressão da MAI e ISI baseado no algoritmo RLS.

\section{Algoritmo LMS no Domínio Transformado, TRLMS}

Os potenciais problemas dos algoritmos RLS e LMS padrão motivaram a busca de algoritmos alternativos. Técnicas no domínio transformado consistem em ortogonalizar o sinal de entrada recebido, aumentando então a velocidade de convergência do algoritmo LMS (MARSHALL; JENKINS; MURPHY, 1989). Uma transformação linear, representada pela matriz $\mathbf{X}$ é aplicada ao sinal de entrada:

$$
\mathbf{r}_{n} \rightarrow \mathbf{X} \mathbf{r}_{n}
$$

objetivando diagonalizar a matriz de correlação, $\mathbf{R}$

$$
\mathbf{R}_{X}=\mathbf{X R X}^{H} \approx \operatorname{diag}\left[\lambda_{1}, \lambda_{2}, \ldots, \lambda_{M}\right]
$$

Com uma matriz de correlação aproximadamente diagonal, o LMS pode usar dimensões de passos ótimas e múltiplas, um para cada derivação do filtro, resultando na seguinte equação de atualização dos coeficientes:

$c(n+1)=c(n)+\mu \operatorname{diag}\left[\hat{\lambda}_{1}, \hat{\lambda}_{2}, \ldots, \hat{\lambda}_{M}\right]^{-1} e^{*}(n) \mathbf{r}(n)$

onde $\hat{\lambda}_{j}=$ estimativa para o $j$-ésimo autovalor de $\mathbf{R}_{\mathbf{X}} ; M=S p$ é a dimensão da linha de atraso com derivações.

A convergência do algoritmo TRLMS é ditada pelo espalhamento dos autovalores da matriz

$$
\operatorname{diag}\left[\hat{\lambda}_{1}, \hat{\lambda}_{2}, \ldots, \hat{\lambda}_{M}\right]^{-1} \mathbf{R}_{X}=\hat{\mathbf{R}}_{X}^{-1} \mathbf{R}_{X} \approx \mathbf{I}_{M}
$$

Idealmente, o espalhamento dos autovalores é agora unitário, e portanto, a velocidade de convergência é muito maior. $\mathrm{O}$ principal problema do emprego da técnica LMS no domínio transformado é escolher uma boa transformação fixa, de modo que seja possível obter aumento na velocidade de convergência que justifique o aumento de complexidade. Este é o caso de canais não estacionários.

\section{Algoritmo Treliça (Lattice) para o Detector MMSE}

O pré-processamento treliça propicia um caminho adaptativo para a realização da ortogonalização buscada na técnica TRLMS, aumentando a convergência ao final do estágio transversal às custas de adaptação adicional de coeficientes provenientes da estrutura treliça. Uma estrutura treliça com coeficientes de reflexão apropriados propicia a ortogonalização de Gram-Schmidt do sinal de entrada. O algoritmo treliça multicanal mostra desempenho e quantidades numéricas muito bons. No entanto, o preço a ser pago é a alta complexidade computacional, da ordem de $\mathcal{O}\left(S p^{3}\right)$.

\section{Algoritmo MMSE Adaptativo Cego}

O maior impedimento no uso do MMSE adaptativo é o requisito da seqüência de treinamento. Isso implica coordenação extra entre receptor e transmissores, bem como um custo adicional no sistema. O tempo é consumido treinando o receptor ao invés do envio de dados úteis. Técnicas cegas podem eliminar a necessidade da seqüência de treinamento. Honig, Madhow and Verdú (HONIG; MADHOW; VERDÚ., 1995) propuseram um método onde o receptor multiusuário cego deve conhecer apenas a seqüência de espalhamento e atraso do usuário de interesse. A resposta impulsiva do receptor adaptativo é decomposta em duas parcelas ortogonais. Para o $k$-ésimo usuário, o vetor representando os coeficientes do filtro no receptor será:

$$
\mathbf{c}_{k}=\mathbf{s}_{k}+\mathbf{x}_{k}
$$


onde $\mathbf{s}_{k}$ é a seqüência de espalhamento que identifica o $k$-ésimo usuário; esta componente não é adaptativa, atuando como âncora no processo de atualização dos coeficientes do filtro; $\mathbf{x}_{k}$ é a componente adaptativa; parcela variável da atualização dos coeficientes; as atualizações restringem-se a serem ortogonais ao vetor âncora.

O critério de otimização adotado busca a mínima energia de saída (MOE). Com isso, pode-se mostrar que os coeficientes do filtro MMSE convergem. Essa abordagem assume que a sequiência de espalhamento do usuário de interesse seja conhecida exatamente no receptor. Na prática, uma estimativa do vetor de espalhamento recebido, $\hat{\mathbf{s}}_{k}$, é empregada como um filtro âncora. Quando houver desajustes, como no caso de distorções no canal, deve-se impor restrições ainda maiores sobre $\mathbf{x}_{k}$. Deve-se permitir ao excesso de energia do filtro receptor, definido por $\chi=\left\|\mathbf{x}_{k}\right\|^{2}$, crescer o suficiente para efetivamente cancelar a MAI.

O algoritmo cego apresenta convergência um pouco mais ruidosa quando comparada à abordagem adaptativa baseada em seqüência de treinamento. Este problema torna-se mais pronunciado quando o desajuste entre a estimativa e o sinal original cresce.

\section{Resultados Numéricos}

Nesta seção são compilados resultados de desempenho para o sistema DS/CDMA, utilizando os algoritmos adaptativos LMS no processo de detecção MuD Descorrelacionador e MMSE. Duas figuras de mérito foram consideradas na obtenção de um quadro comparativo entre as duas estratégias $\mathrm{MuD}$, convergência e taxa de erro de bit, resultando em três tipos de gráficos de desempenho:

- MSE normalizado $\times$ número de iterações até a convergência;
- $\mathrm{BER} \times E_{b} / N_{0}$;

- BER $\times$ carregamento do sistema

Para os dois primeiros tipos de gráfico de desempenho foram adotados os seguintes parâmetros: sequiências aleatórias de comprimento $N=31$, número de usuários $K=10$ usuários, resultando em um carregamento $L=0,32$ e cenários com controle perfeito de potência. Para o terceiro tipo de gráfico de desempenho foram adotados $N=31$, região de relação sinal-ruído média $\left(E_{b} / N_{0}=7 \mathrm{~dB}\right)$ e as seguintes condições de carregamento: $L=$ $1 / 31,5 / 31,10 / 31,15 / 31,20 / 31,25 / 31$ e $31 / 31$.

Em todas as simulações Monte Carlo (MCS), adotou-se um número mínimo de erros/ponto $=20$. Para efeito de comparação, foram incluídos os desempenhos dos detectores convencional (CD), dos detectores Descorrelacionador e MMSE fixos e o limite quando há apenas um único usuário ativo no sistema ( $\mathrm{SuB}$ - single user bound) com modulação BPSK e canal AWGN (PROAKIS, 1989).

Os valores adotados para os parâmetros dos algoritmos adaptativos foram obtidos em duas etapas, considerando carregamento fixo $L=10 / 31$ :

1. simulações preliminares foram conduzidas adotando-se valores típicos encontrados na literatura;

2. simulações adicionais foram feitas visando à otimização dos parâmetros, de modo não exaustivo, porém com resultados de desempenho superiores aos obtidos na primeira etapa.

As figuras 4 a 12 sintetizam os principais resultados de simulação MCS em canal AWGN síncrono. Observe-se que, como esperado, à medida que a relação sinal-ruído cresce, o erro quadrático médio 
decresce para os três tipos de algoritmos adaptativos analisados: LMS Descorrelacionador, Fig. 4, LMS MMSE single-user, Fig. 5 e LMS MMSE multiusuário, Fig. 6.

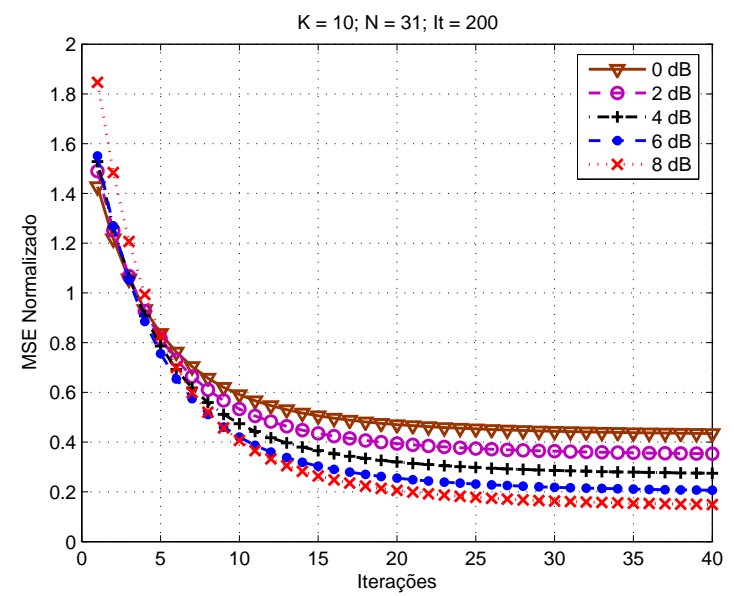

Figura 4: Convergência do algoritmo adaptativo LMS Descorrelacionador para diferentes $E_{b} / N_{0}$.

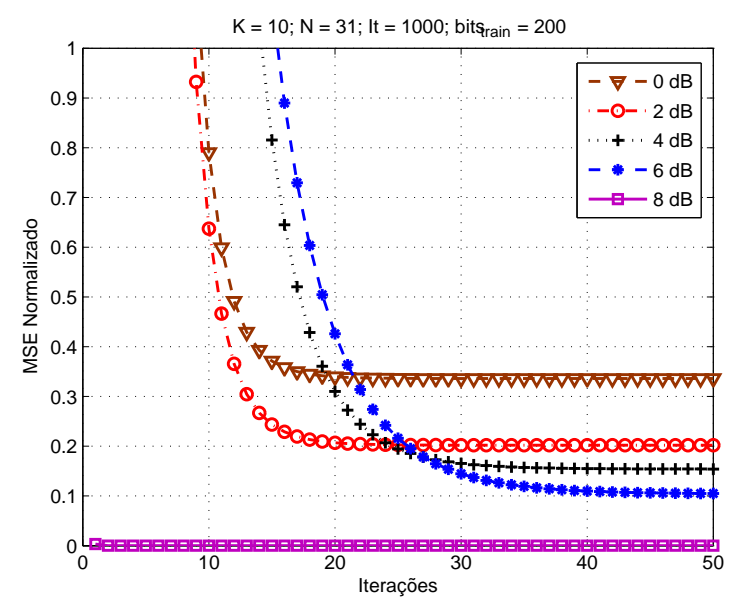

Figura 5: Convergência do algoritmo adaptativo LMS MMSE single-user para diferentes $E_{b} / N_{0}$.

Para todas as figuras de mérito consideradas, o algoritmo adaptativo LMS Descorrelacionador mostrou ter desempenho semelhante ao Descorrelacionador fixo (DEC), porém melhor do que o Convencional, para carregamentos de até $L \approx 0,7$, Figs. 7 e 8. Observe-se que melhoria adicional no desempenho do algoritmo adaptativo LMS Descorrelacionador da Fig. 8 poderia ser obtida caso os parâmetros

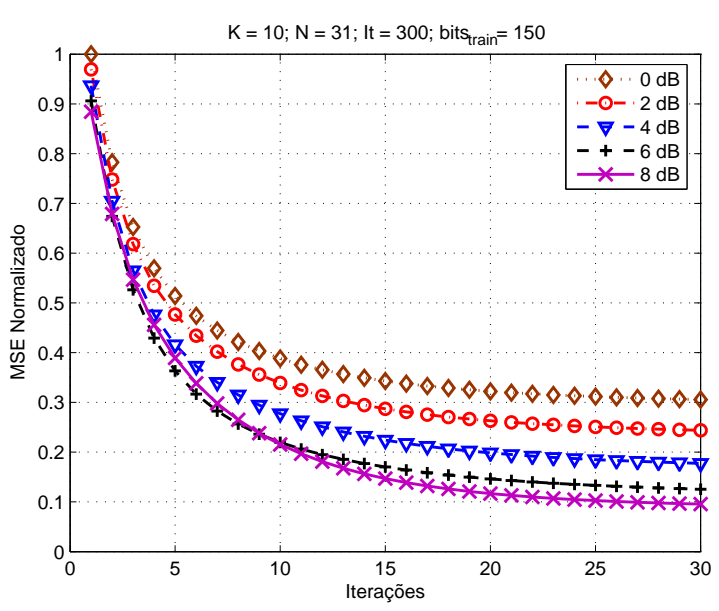

Figura 6: Convergência do algoritmo adaptativo LMS MMSE multiusuário para diferentes $E_{b} / N_{0}$.

do algoritmo também fosse otimizado em função do aumento do carregamento.

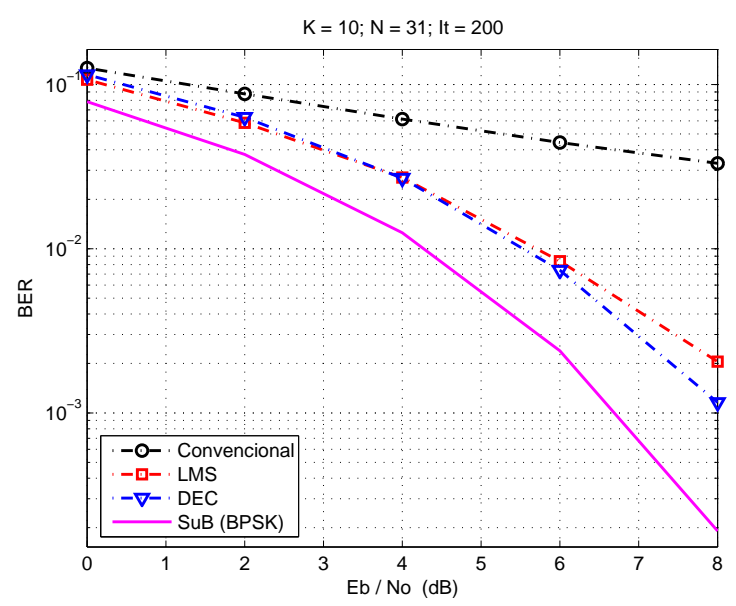

Figura 7: BER para o algoritmo adaptativo LMS Descorrelacionador

O algoritmo adaptativo LMS MMSE single-user apresentou melhor desempenho em relação ao detector Convencional e semelhante ao MMSE fixo, principalmente na região de médio carregamento, Fig. 9 e 10. Já para carregamentos próximos à unidade, o LMS MMSE single-user adaptativo apresenta melhor desempenho em relação ao MMSE fixo. No entanto, para a região de elevado $E_{b} / N_{0}$ e baixos carregamentos, o algoritmo MMSE fixo apresentará uma tendência de melhor desempenho em relação ao LMS MMSE single-user adaptativo, Fig. 9. 


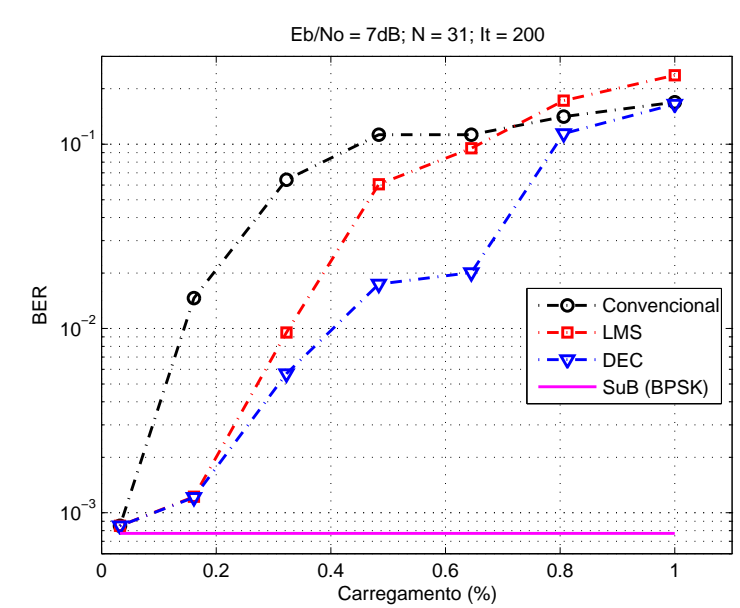

Figura 8: BER para o algoritmo adaptativo LMS Descorrelacionador em função do aumento de carregamento

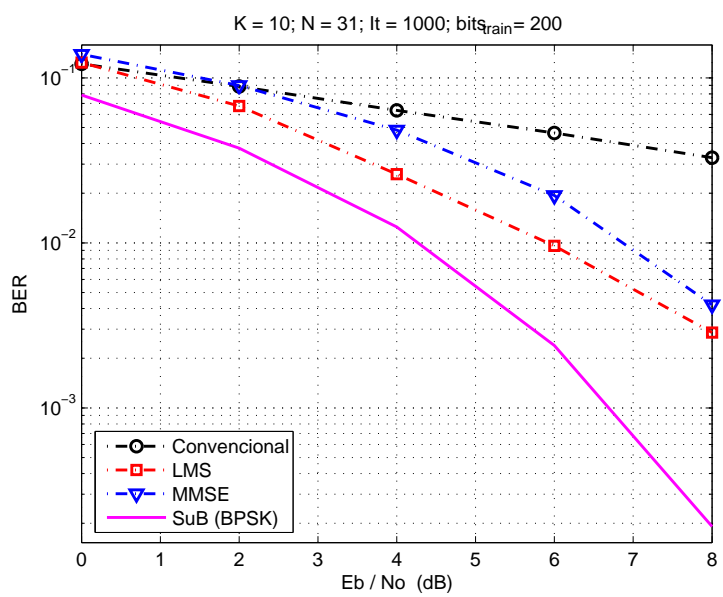

Figura 9: BER para o algoritmo adaptativo LMS MMSE single-user

Finalmente, a aplicação do algoritmo adaptativo LMS MMSE multiusuário resultou em desempenho semelhante ao MMSE fixo para a faixa de carregamento $0<L \lesssim 0,5$ e superior ao detector Convencional para uma faixa de carregamento $0<L \lesssim$ 0,6 , Figs. 11 e 12 . Com a elevação de $E_{b} / N_{0}$ e mantido o carregamento em níveis moderados, esta diferença de desempenho tende a ser maior também em relação ao MMSE fixo, Fig. 11. Novamente, caso os parâmetros do algoritmo LMS MMSE multiusuário adaptativo fossem otimizados em função do número de usuários ativos, seu desempenho tornar-

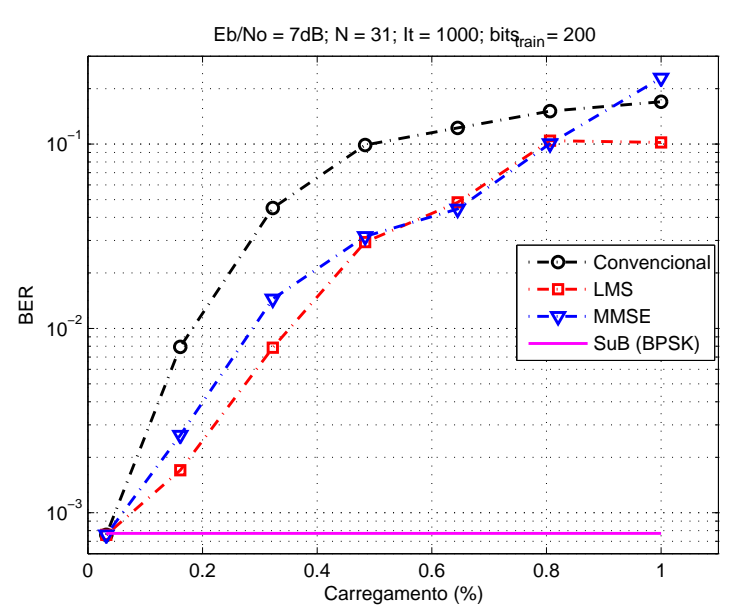

Figura 10: BER para o algoritmo adaptativo LMS MMSE single-user em função do aumento de carregamento

se-ia semelhante ao MMSE fixo multiusuário para toda a faixa de carregamentos, $0 \leq L \leq 1$.

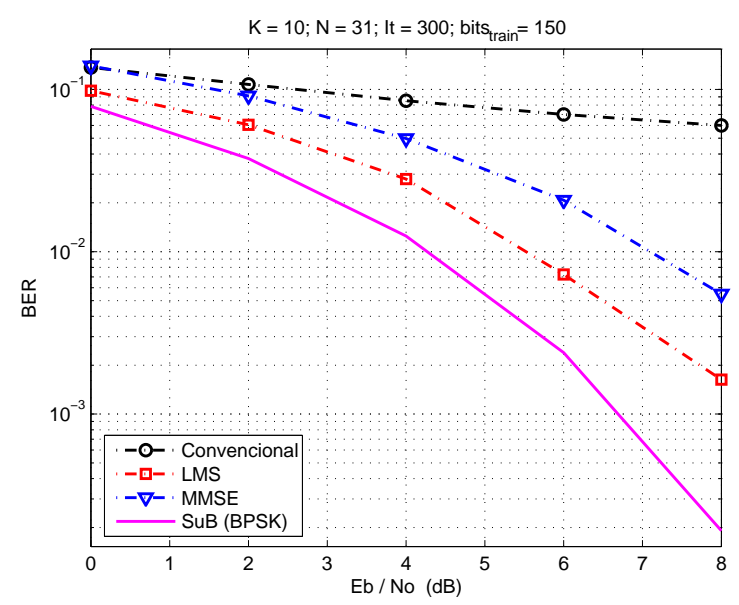

Figura 11: BER para o algoritmo adaptativo LMS MMSE multiusuário

\section{Conclusões}

Este trabalho de revisão mostrou o potencial incremento na capacidade dos sistemas de comunicação associado aos receptores multiusuários adaptativos. Diferentemente da detecção convencional com filtro casado single-user, a detecção multiusuário utiliza as informações dos demais usuários ativos para 


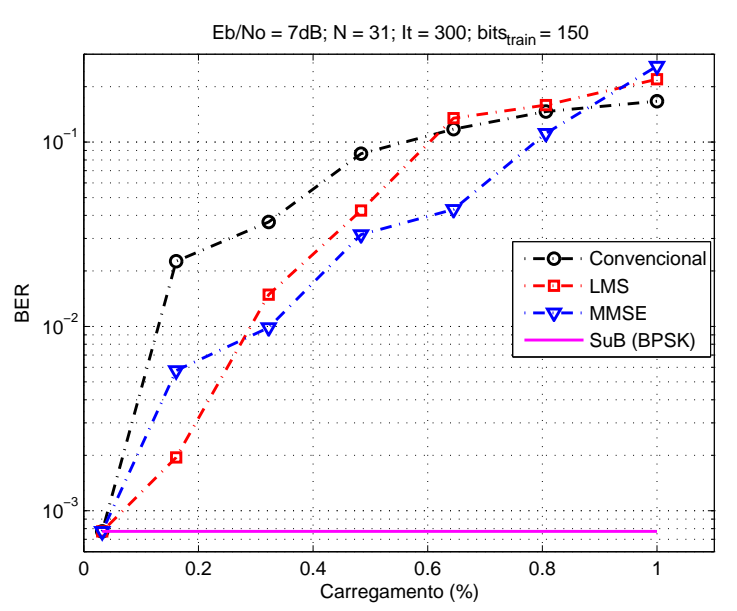

Figura 12: BER para o algoritmo adaptativo LMS MMSE multiusuário em função do aumento de carregamento

anular tais sinais que aparecem como interferência MAI quando da deteç̧ão do usuário de interesse. Em contrapartida, ter-se-á um aumento na complexidade de implementação dos sistemas. O receptor DS-CDMA Convencional é extremamente sensível ao efeito near-far, requerendo grandes cuidados com controle de potência e o projeto das seqüências de espalhamento a fim de obter aceitável desempenho, além de apresentar capacidade de sistema bem abaixo da capacidade de canal. Isto tem motivado pesquisas de algoritmos de detecção multiusuário, objetivando reduzir ou mesmo eliminar a MAI.

O receptor MMSE adaptativo permite operar como um receptor single-user, não necessitando conhecimento explícito dos usuários interferentes, sendo capaz de prover supressão da MAI. Permite recepção descentralizada, importante vantagem em termos de implementação. O Descorrelacionador adaptativo pode ser atrativo em aplicações centralizadas, onde seja possível obter razoável conhecimento dos parâmetros dos usuários interferentes.

A implementação do filtro LMS é muito usado em MuD DS/CDMA adaptativo devido à sua baixa complexidade computacional e boa estabili- dade numérica. O tempo de convergência do algoritmo LMS MuD deve ser compatível com o período da informação transmitida e uma redução na taxa líquida de dados do sistema deve ser prevista no caso dos algoritmos adaptativos que requerem símbolos de treinamento no detector. Finalmente, a implementação de algoritmos adaptativos cegos esbarram no problema de compatibilizarem velocidade de convergência e recuperação de desvanecimentos profundos, no caso de canais multiplicativos (LIM; GONG; FARHANG-BOROUJENY, 1999).

Questões importantes de pesquisa e ainda remanescentes incluem: a garantia de adequado desempenho para algoritmos adaptativos; o aumento das taxas de convergência; a melhoria de desempenho dos algoritmos cegos.

\section{Referências}

ABRÃO, T.; JESZENSKY, P. J. E. Detectores multiusuários para ds-cdma - parte i: Lineares fixos. Revista da Sociedade Brasileira de Telecomunicações, SBrT, v. 16, n. 2, p. 122-137, Dezembro 2001.

ABRÃO, T.; JESZENSKY, P. J. E. Detectores multiusuários para ds-cdma - parte ii: Cancelamento de interferência. Revista da Sociedade Brasileira de Telecomunicações, SBrT, v. 16, n. 2, p. 138-156, Dezembro 2001.

CHEN, D.; ROY., S. An adaptive multiuser receiver for cdma systems. IEEE Journal on Selected Areas in Communications, v. 12, n. 5, p. 808-16, June 1994.

HAYKIN, S. Adaptive Filter Theory. 3rd. ed. [S.1.]: Prentice Hall, 1996. (ISBN: 0-13-322760-X). 989 pages.

HONIG, M. L.; MADHOW, U.; VERDÚ., S. Blind adaptive multiuser detection. IEEE Transactions on Information Theory, v. 41, n. 4, p. 944-60, July 1995.

KARAYIANNIS, N.; CHOOKIARTI, J. Directly estimated adaptive detectors for code-division multiple-access signals. IEEE Transactions on Communications, v. 53, n. 2, p. 356-365, February 2005.

LI, M.; HAMOUDA, W. Adaptive multistage detection for ds-cdma systems in multipath fading channels. In: 61ST VEHICULAR TECHNOLOGY CONFERENCE. [S.1.]: IEEE, 2005. v. 1, p. 300-304. 
LIM, T. J.; GONG, Y.; FARHANG-BOROUJENY, B. Convergence analysis of lms multiuser cdma detectors. In: WIRELESS COMMUNICATIONS AND NETWORKING CONFERENCE. [S.1.]: IEEE, 1999. v. 1, p. 75-79.

LIN, G.-J.; LEE, T.-S.; TAN, C.-C. Design of a low complexity partially adaptive cdma receiver using conjugate gradient technique. In: GLOBAL TELECOMMUN. CONF. [S.1.]: IEEE, 2002. v. 1, p. 443-447.

MADHOW, U. Blind adaptive interference suppression for direct-sequence cdma. Proceedings of the IEEE, v. 86, n. 10, p. 2049-69, October 1998.

MAROUANE, H.; KACHOURI, A.; KAMOUN, L. Lms adaptive filter single-user receiver. In: THE 16TH INTERNATIONAL CONFERENCE ON MICROELECTRONICS. [S.1.]: IEEE, 2004. p. 637-641.

MARSHALL, D.; JENKINS, W.; MURPHY, J. The use of orthogonal transforms for improving performance of adaptive filters. IEEE Trans. Circuits Systems, v. 36, n. 4, p. 474-84, April 1989.

MILLER, S. L. An adaptive DS-CDMA receiver for multiuser interference rejection. IEEE Transactions on Communications, v. 43, n. 2/3/4, p. 1746-55, Feb./March/April 1995.

MOSHAVI, S. Multi-user detection for DS-CDMA communications. IEEE Communications Magazine, p. 12436, October 1996.

MUCCHI, L.; MOROSI, S.; RE, E. D.; FANTACCI, R. A new algorithm for blind adaptive multiuser detection in frequency selective multipath fading channel. IEEE Transactions on Wireless Communications, v. 3, n. 1, p. 235247, January 2004.

MYERS, T.; MAGANA, M. E. An adaptive implementation of "one-shot" decorrelating detector for CDMA communications. IEEE Transactions Circuits Systems II, v. 44, p. 762-5, September 1997.

NIEKERK, B. van; MNENEY, S. Blind iterative mud with error coding. In: 7TH CONFERENCE IN AFRICA. [S.1.]: IEEE, 2004. v. 1, p. 179-184.

PROAKIS, J. Digital Communications. second edition. [S.l.]: McGraw-Hill, 1989.

SLOCK, D. T. M. On the convergence behavior of the lms and the normalized $\mathrm{lms}$ algorithms. IEEE Transactions on Signal Processing, v. 41, n. 9, p. 2811-2825, September 1993.

SUN, L.; BI, G.; ZHANG, L. Fast converging blind adaptive multiuser detectors with linearly constrained cma for ds/cdma systems. In: EIGHTH INTERNATIONAL SYMPOSIUM ON SPREAD SPECTRUM TECHNIQUES AND APPLICATIONS. Sydney: IEEE, 2004. p. 1749-1758.
TAKAWIRA, J. W. F. Performance analysis of the linearly constrained constant modulus algorithm-based multiuser detector. IEEE Transactions on Signal Processing, v. 53, n. 2, p. 643-653, February 2005. Part 1.

TONG, L.; PERREAU, S. Multichannel blind identification: From subspace to maximum likehood methods. Proceedings of the IEEE, v. 10, n.86, p. 1951-68, October 1998.

UENG, F.-B.; JENG, L.-D. Adaptive cdma receiver for downlink multiuser interference suppression. IEE Electronics Letters, v. 39, n. 6, p. 552-553, March 2003.

ULUKUS, S.; YATES, R. D. Blind adaptive decorrelating detector for CDMA systems. IEEE Journal on Selected Areas in Communications, v. 16, n.8, p. 1530-41, October 1998.

VERDÚ, S. Multiuser Detection. [S.1.]: Cambridge University Press, 1998.

VUCETIC, B.; WOODWARD, G. Adaptive detection for ds-cdma. Proceedings of the IEEE, v. 86, n. 7, p. 1413-35, July 1998.

WANG, X.; POOR, V. Blind equalization and multiuser detection in dispersive CDMA channels. IEEE Transactions on Communications, v. 46, n.12, p. 91-103, January 1998.

WANG, X.; POOR, V. Blind multiuser detection: A subspace approach. IEEE Transactions on Information Theory, v. 44, n.2, p. 677-90, March 1998.

XIE, L.; SHORT, T. S.; RUSHFORTH, C. K. A family of suboptimum detector for coherent multiuser communication. IEEE Journal on Selected Areas in Communications, v. 8, n.4, p. 683-90, May 1990.

ZHANG, W.; D'AMOURS, C.; YONGACOGLU, A. Adaptive soft-input soft-output multiuser detection for asynchronous coded ds-cdma systems. In: WIRELESS COMMUNICATIONS AND NETWORKING CONFERENCE. [S.1.]: IEEE, 2005. v. 1, p. 227-232.

ZHU, S.; ARASARATNAM, P.; CONSTANTINIDES, A. Blind adaptive cdma receivers based on independent component separation with user-and-delay identification. In: 6TH INTERNATIONAL CONFERENCE ON SIGNAL PROCESSING. [S.1.]: IEEE, 2002. v. 2, p. 1275-1278.

ZVONAR, Z.; DUEL-HALLEN, A. Multiuser detection for cdma systems. IEEE Personal Communications, p. 46-58, April 1995. 